\title{
A solution to the cosmic ray anisotropy problem
}

\author{
P. Mertsch ${ }^{1}$ and S. Funk ${ }^{2}$ \\ ${ }^{1}$ Kavli Institute for Particle Astrophysics \& Cosmology, 2575 Sand Hill Road, M/S 29, \\ Menlo Park, CA 94025, USA \\ ${ }^{2}$ Erlangen Center for Astroparticle Physics (ECAP), Friedrich-Alexander Universität Erlangen-Nürnberg, \\ Erlangen, Germany \\ Correspondence to: P. Mertsch (pmertsch@stanford.edu)
}

Received: 28 May 2015 - Revised: 12 August 2015 - Accepted: 9 September 2015 - Published: 8 October 2015

\begin{abstract}
Observations of the cosmic ray (CR) anisotropy are widely advertised as a means of finding nearby sources. This idea has recently gained currency after the discovery of a rise in the positron fraction and is the goal of current experimental efforts, e.g., with AMS-02 on the International Space Station. Yet, even the anisotropy observed for hadronic CRs is not understood, in the sense that isotropic diffusion models overpredict the dipole anisotropy in the TeV-PeV range by almost two orders of magnitude. Here, we consider two additional effects normally not considered in isotropic diffusion models: anisotropic diffusion due to the presence of a background magnetic field and intermittency effects of the turbulent magnetic fields. We numerically explore these effect by tracking test-particles through individual realisations of the turbulent field. We conclude that a large misalignment between the CR gradient and the background field can explain the observed low level of anisotropy.
\end{abstract}

\section{Introduction}

Information on sources and transport of cosmic rays (CRs) are gained from three types of observations: spectra, composition and anisotropies. Among those three, anisotropies have a somewhat paradoxical status: on the one hand the observed arrival directions of CRs posses a low level of anisotropy - about 1 part in 1000 or 10000 at TeV-PeV energies. Given the likely inhomogeneous distribution of sources in the Galaxy, this requires an efficient mechanism to randomise the directions of CRs and therefore constrains models of CR transport. On the other hand, a residual anisotropy in the arrival direction encodes information about the position and age of sources. This has been considered as a possibility for finding nearby sources, in particular in the context of the observed rise in the positron fraction. We note that this situation is somewhat reminiscent of the cosmic microwave background (CMB): while the angular power spectrum of anisotropies contains information on the parameters of the cosmological model, the high level of isotropy requires a mechanism that allows far away regions to be in causal contact at early times.
It has been known for a long time that pitch-angle scattering mediated by resonant interaction between CRs and turbulent magnetic fields can provide the necessary randomisation (as reviewed by, e.g., Ginzburg et al., 1990). Pitch-angle scattering is also mediating spatial diffusion and if the distribution of sources is asymmetric with respect to the observer, e.g. there are more sources towards the Galactic centre than towards the anti-centre, a small degree of residual anisotropy is expected. The amplitude $a$ of the dipole in the arrival directions, defined as the relative difference of observed maximum and minimum fluxes, $\phi_{\max }$ and $\phi_{\min }$, is related to the gradient in the isotropic part $f_{0}$ of the distribution function $f(\boldsymbol{x}, p, \mu)=f_{0}(\boldsymbol{x}, p)(1+a \mu)$,

$a=\frac{\phi_{\max }-\phi_{\min }}{\phi_{\max }+\phi_{\min }} \simeq \frac{3 D}{v} \frac{\left|\nabla f_{0}\right|}{f_{0}}$

where $D$ is the isotropic diffusion coefficient and $v$ the particle speed.

Predictions for the dipole anisotropy start by assuming a spatial distribution of sources, e.g., from supernova remnant or pulsar surveys. From the propagated CR distributions, the dipole amplitude is then computed as a function of energy. 
This picture, however, does not compare well with measurements (Antoni et al., 2003; Gerhardy et al., 1984; Kifune et al., 1985; Nagashima et al., 1990; Aglietta et al., 1996, 2003; Amenomori et al., 2005; Abdo et al., 2009; Aglietta, 2009; Abbasi, 2012; Aartsen et al., 2013). As shown in Fig. 1, a prediction from the isotropic diffusion model (dotted line, cf. Blasi and Amato, 2012) is almost two orders of magnitude higher than the measurements between $100 \mathrm{TeV}$ and $1 \mathrm{PeV}$. This discrepancy has come to be known as the cosmic ray anisotropy problem (Hillas, 2005; Ptuskin et al., 2006; Pohl et al., 2012; Evoli et al., 2012). Nevertheless, using the observed distribution of arrival directions, in particular the dipole has been widely advertised as a means to infer the presence of young, nearby sources (Buesching et al., 2008; Sveshnikova, 2013; DiBernardo et al., 2011; Borriello et al., 2010; Linden and Profumo, 2013).

In the following we suggest two modifications to the theoretical picture that in combination can decrease the predicted anisotropy and bring it into agreement with observations. First, in the presence of a background magnetic field $\boldsymbol{B}_{0}$ and for low levels of turbulence - defined as the ratio of turbulent energy density and total energy density, $\eta=\delta B^{2} /\left(B_{0}^{2}+\delta B^{2}\right)$ - diffusion is inherently anisotropic, with the diffusion along $\boldsymbol{B}_{0}$ much more efficient than perpendicular to it. Instead of Eq. (1), the dipole amplitude should read (see, e.g. Ginzburg et al., 1990)

$$
a=\frac{\frac{3}{2} \int_{-1}^{1} \mathrm{~d} \mu \mu f(\mu)}{f_{0}}=\frac{3}{v} \frac{\left|\partial f_{0} / \partial x\right|}{f_{0}} D_{\|} .
$$

Note that now the amplitude depends on the parallel diffusion coefficient $D_{\|}$and the CR gradient along $\boldsymbol{B}_{0}$ (assumed to be in the $x$ direction). This introduces a new degree of freedom, the angle between $\boldsymbol{B}_{0}$ and the CR gradient direction $\nabla f_{0} /\left|\nabla f_{0}\right|$.

Second, the intermittency in the turbulent magnetic field can play a role. Analytical computations of the transport of CRs can only predict the average distribution function $f$ for an ensemble of turbulent magnetic fields. Due to the stochastic nature of the turbulent fields, in one particular realisation of the turbulent magnetic field we expect deviations from the ensemble average and consequently also deviations of the dipole direction and amplitude from the ensemble averaged direction and amplitude. We note that such intermittency effects have also been argued to be the cause of the CR small scale anisotropy observed at TeV-PeV energies (Giacinti and Sigl, 2011; Ahlers, 2013; Ahlers and Mertsch, 2015).

\section{Methodology}

We explore both these effects, i.e., the anisotropic diffusion and turbulence intermittency effects, by numerically back-tracking particles through individual realisations of a turbulent magnetic field. Given a large number of trajec-

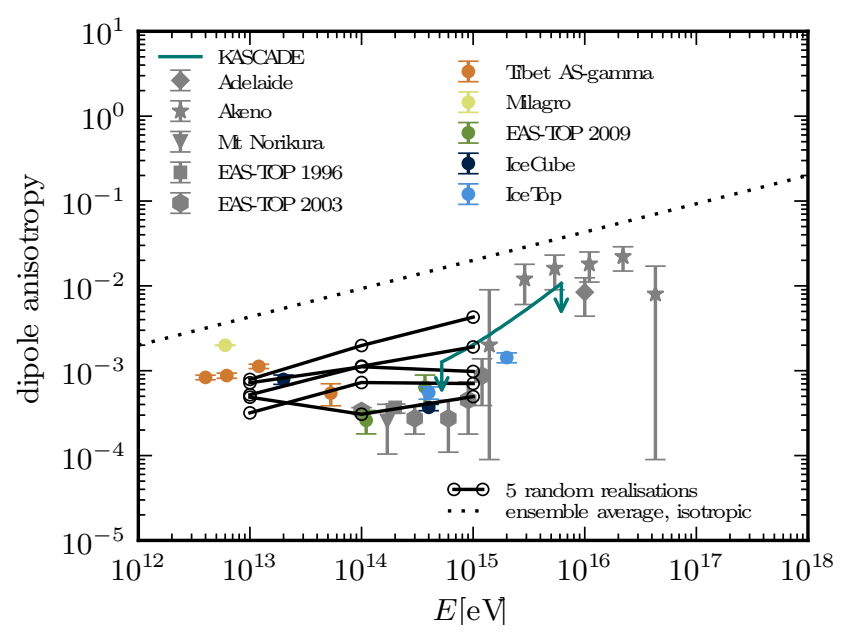

Figure 1. Comparison between measurements of the dipole amplitude (Antoni et al., 2003; Gerhardy et al., 1984; Kifune et al., 1985; Nagashima et al., 1990; Aglietta et al., 1996, 2003; Amenomori et al., 2005; Abdo et al., 2009; Aglietta, 2009; Abbasi, 2012; Aartsen et al., 2013) and models: the dotted line shows the prediction from an isotropic diffusion model (Blasi and Amato, 2012), the open black circles show the dipole anisotropy in five random realisations of the turbulent magnetic field for an angle between CR gradient and background magnetic field close to $90^{\circ}$.

tories, $\left\{\boldsymbol{x}_{i}(t), \boldsymbol{p}_{i}(t)\right\}$, we can compute the arrival directions seen by an observer at position $\boldsymbol{x}_{\mathrm{obs}}$ and time $t_{0}$ from an assumed quasi-stationary CR distribution at an earlier time $\left(t_{0}-\Delta t\right)$ and exploiting Liouville's theorem: $f\left(\boldsymbol{x}_{\mathrm{obs}}\right.$, $\left.\boldsymbol{p}_{i}\left(t_{0}\right)\right)=f\left(\boldsymbol{x}_{i}\left(t_{0}-\Delta t\right), \boldsymbol{p}_{i}\left(t_{0}-\Delta t\right)\right)$. Note the intermittency effect is due to the local configuration of the turbulent magnetic field, i.e., the arrival directions can only reflect the turbulent field over the last few scattering lengths. This justifies truncating the expansion of the spatial distribution after the first derivative, i.e., the CR gradient $\nabla f_{0}$. Therefore, we adopt the quasi-stationary distribution $f(x$, $\left.t_{0}-\Delta t\right)=f_{0}+x \partial f_{0} / \partial x$. Note that in general there is an angle between the gradient direction $\nabla f_{0} /\left|\nabla f_{0}\right|$ and $\boldsymbol{B}_{0}$.

We solve the relativistic equations of motions with a 5th order adaptive Runge-Kutta algorithm (Sutherland et al., 2010) at three energies, 10, 100 and 1000 TV. For the level of turbulence we consider $\eta=1$ and 0.1 which should bracket its uncertainty. To bridge the large dynamical range between the particle gyroradius and a few times its scattering length, we set up the turbulent magnetic field on a set of nested grids (Giacinti et al., 2011), assuming a Kolmogorov spectrum, an outer scale $L=100 \mathrm{pc}$ and a total RMS field strength of $4 \mu \mathrm{G}$.

\section{Results}

In Fig. 2 we show the dipole directions in 50 realisations of the turbulent magnetic field in the absence of $\boldsymbol{B}_{0}$ for $1 \mathrm{PeV}$ CRs. The position and direction of each circle shows the dipole direction and amplitude in one random realisation, re- 


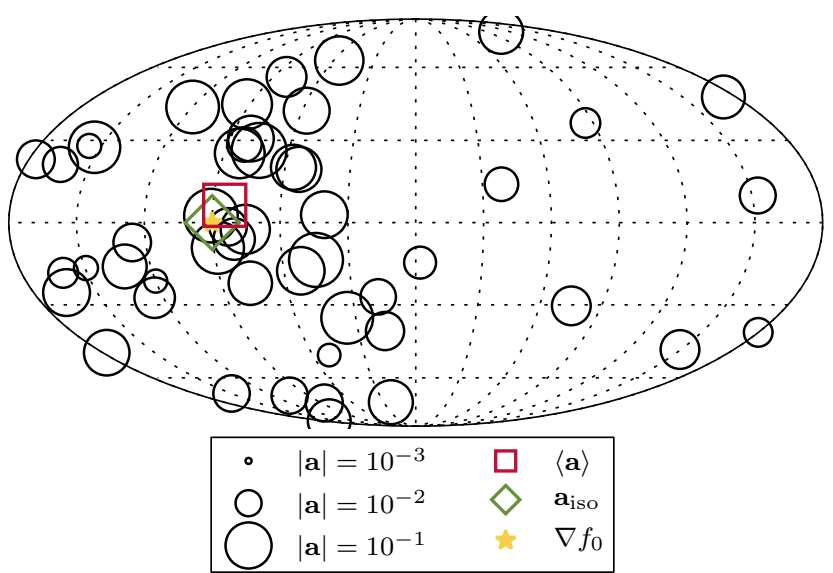

Figure 2. Dipole directions (centres of circles) and amplitudes (radii of circles) in 50 random realisations of the local turbulent magnetic field at $1 \mathrm{PV}$ and for $\eta=1$. The CR gradient is denoted by the yellow star, the prediction from an isotropic diffusion model by the green diamond and the average of the 50 random realisations by the red square.

spectively. Two things are noteworthy: It is clear that the individual dipole directions are in general not pointing in the $\mathrm{CR}$ gradient direction (denoted by the yellow star). Second, there is some scatter also in the amplitude of individual dipoles. The (vectorial) mean, however, shown by the red square, is in good agreement both with the expected direction and amplitude. For all energies considered, the ensemble averaged dipole amplitude reproduces the result of Blasi and Amato (2012), cf. dotted line in Fig. 1. We further explore the scatter in the amplitude in Fig. 3 that shows the distribution of dipole amplitudes as a function of the longitude of the CR gradient direction. (As there is no other direction in this setup, we would not expect any systematic dependence on this direction which is in fact the case.) The dipole amplitudes scatter by a factor of a few around the expectation value $|\langle\boldsymbol{a}\rangle|$ that is always in agreement with the prediction from the isotropic diffusion model, $\left|\boldsymbol{a}_{\text {iso }}\right|$. Still, the predictions are at least an order of magnitude above the upper limit from KASCADE.

Figure 4 shows the distribution of dipole directions and amplitudes in the presence of $\boldsymbol{B}_{0}$ with $\eta=0.1$, i.e., a weak turbulent field, and assuming an angle of $\sim 90^{\circ}$ between $\nabla f_{0}$ and $\boldsymbol{B}_{0}$. The amplitudes are significantly suppressed and the directions show again a large scatter, but now cluster around the $\boldsymbol{B}_{0}$ direction, indicated by the blue cross, which is also the direction of the ensemble average dipole. From Fig. 5, it can be seen how the distribution of dipole amplitudes depends on the angle between $\boldsymbol{B}_{0}$ and $\boldsymbol{\nabla} f_{0}$ : for small angles, the amplitudes closely track the prediction from the isotropic diffusion model, with relatively little scatter. At angles close to $90^{\circ}$ though, the amplitude can be significantly suppressed, at the minimum limited only by the level of turbulence $\eta$. For the adopted $\eta=0.1$, the mean amplitude is in agreement with KASCADE. We also note that the scatter is enhanced

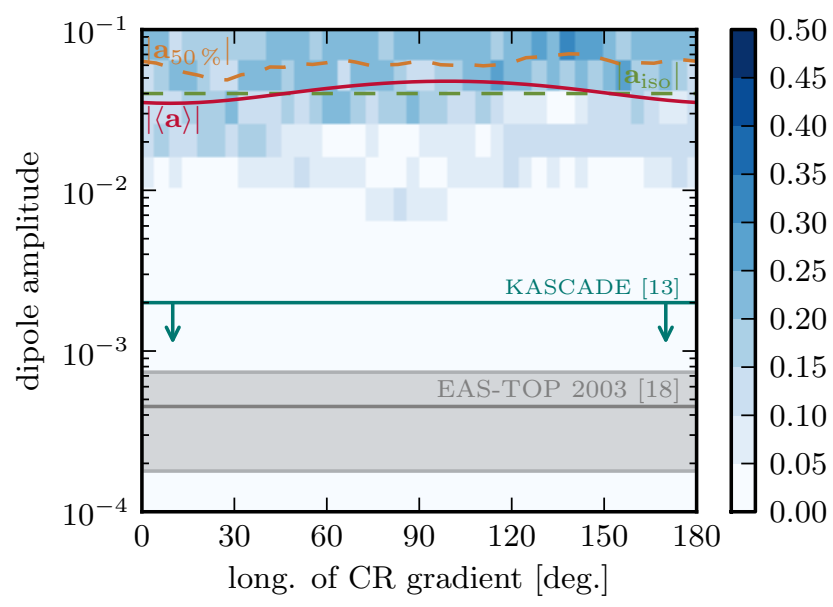

Figure 3. Distribution of dipole amplitudes at $1 \mathrm{PV}$ and for $\eta=1$ as a function of the longitude of the CR gradient. Each vertical slice is normalised to one. The orange dashed, red solid and green dashed lines show the median, the amplitude of the vectorial mean and the prediction for isotropic diffusion, respectively. The KASCADE upper limit and EAS-TOP measurement at $\sim 1 \mathrm{PeV}$ are denoted by the cyan solid line and grey band, respectively.

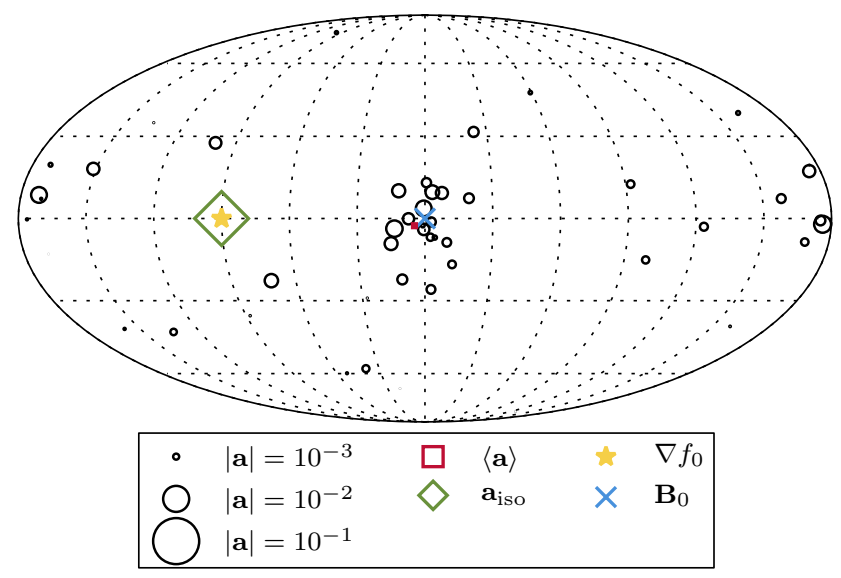

Figure 4. Same as Fig. 2, but with a non-zero regular field $(\eta=0.1)$, indicated by the blue cross.

for angles around $90^{\circ}$. About $20 \%$ of the turbulent field realisations lead to a dipole amplitude that is compatible with the EAS-TOP measurement.

We now turn back to Fig. 1 which also shows the dipole amplitudes for 5 random realisations of the turbulent magnetic field (open circles connected by solid lines). It is interesting to see that not all configurations show the monotonous behaviour with energy of the ensemble average. One configuration in particular shows a falling amplitude between 10 and $100 \mathrm{TeV}$ before rising again - very much like the data. 


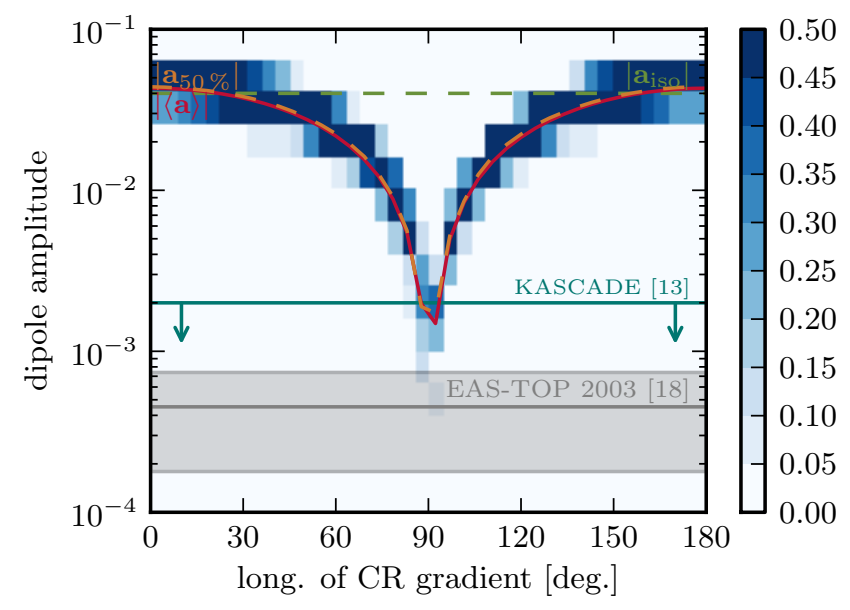

Figure 5. Same as Fig. 3, but with a non-zero regular field $(\eta=0.1)$. The ensemble average $|\langle a\rangle|$ follows the prediction from anisotropic diffusion, $|\langle a\rangle| \propto \sqrt{\left(v / \Omega_{0}\right)^{2}+\cos ^{2} \psi}$ where $v, \Omega_{0}$ and $\psi$ are the scattering rate, gyrofrequency and angle between $\boldsymbol{B}_{0}$ and $\nabla f_{0}$ (i.e., the longitude of the CR gradient in our setup), respectively.

\section{Conclusions}

Observations of the dipole direction in the arrival distribution of CRs have been promoted as a means of source searches, yet the measured dipole amplitude is systematically overpredicted by isotropic diffusion models. We suggest two effects, that can resolve this so-called anisotropy problem: the anisotropic nature of diffusion in the presence of a background magnetic field and intermittency, that is fluctuations of the distribution function across individual realisations of the turbulent field. While the conjunction of both effects can sufficiently suppress the dipole amplitude (for small turbulence level $\eta$ and if the background field and CR gradient are close to perpendicular), this is also casting doubt on the chances of finding sources in the dipole direction: in the high turbulence level case $(\eta=1)$ the directions scatter significantly around the CR gradient direction (see Fig. 2). In the low turbulence case $(\eta=0.1)$, there is also scatter and in general the dipole directions cluster around the field direction, not the gradient direction. For the preferred case with a large angle between gradient and field, see Fig. 3, the scatter is so large that not even the field direction can be inferred.

Acknowledgements. We would like to thank Markus Ahlers for stimulating discussions. P. Mertsch is supported by DoE contract DE-AC02-76SF00515 and a KIPAC Kavli Fellowship. We acknowledge use of the HEALP ix package (Gorski et al., 2005).

Edited by: P. Desiati

Reviewed by: M. Ahlers and L. Drury

\section{References}

Aartsen, M. G., Abbasi, R., Abdou, Y., et al. (IceCube Collaboration): Observation of Cosmic Ray Anisotropy with the IceTop Air Shower Array, Astrophys. J., 765, 55, 2013.

Abbasi, R., Abdou, Y., Abu-Zayyad, T., et al. (IceCube Collaboration): Observation of an Anisotropy in the Galactic Cosmic Ray arrival direction at $400 \mathrm{TeV}$ with IceCube, Astrophys. J., 746, 33, 2012.

Abdo, A. A., Allen, B. T., Aune, T., Berley, D., Casanova, S., Chen, C., Dingus, B. L., and Ellsworth, R. W.: The Large Scale CosmicRay Anisotropy as Observed with Milagro, Astrophys. J., 698, 2121-2130, 2009,

Aglietta, M., Alessandro, B., Antonioli, P., Arneodo, F., Bergamasco, L., Bertaina, M., Bosio, A., Castellina, A., Castagnoli, C., Chiavassa, A., Cini Castagnoli, G., D’Ettorre Piazzoli, B., di Sciascio, G., Fulgione, W., Galeotti, P., Ghia, P. L., Iacovacci, M., Mannocchi, G., Melagrana, C., Mengotti Silva, N., Morello, C., Navarra, G., Riccati, L., Saavedra, O., Trinchero, G. C., Vallania, P., Vernetto, S., and EAS-Top Collaboration: A Measurement of the solar and sidereal cosmic ray anisotropy at $E(0)$ approximates 10**14-eV, Astrophys. J., 470, 501-505, 1996.

Aglietta, M., Alekseenko, V. V., Alessandro, B., Antonioli, P., Arneodo, F., Bergamasco, L., Bertaina, M., Bonino, R., Castellina, A., Chiavassa, A., D’Ettorre Piazzoli, B., Di Sciascio, G., Fulgione, W., Galeotti, P., Ghia, P. L., Iacovacci, M., Mannocchi, G., Morello, C., Navarra, G., Saavedra, O., Stamerra, A., Trinchero, G. C., Valchierotti, S., Vallania, P., Vernetto, S., and Vigorito, C.: Evolution of the cosmic ray anisotropy above $10^{14} \mathrm{eV}$, Astrophys. J., 692, L130-L133, 2009.

Aglietta, M., Alessandron, B., Antonioli, P., Arneodo, F., Bergamasco, L., Bertaina, M., Castagnoli, C., Castellina, A., Chiavassa, A., Castagnoli, G. C., D'Ettore Piazzoli, B., Di Sciascio, G., Fulgione, W., Galeotti, P., Ghia, P. L., Iacovacci, M., Mannocchi, G., Morello, C., Navarra, G., Saavedra, O., Trinchero, G. C., Valchierotti, S., Vallania, P., Vernetto, S., Vigorito, C., and EASTOP Collaboration: The cosmic ray anisotropy between $10^{* * 14}$ $\mathrm{eV}$ and $10 * * 15-\mathrm{eV}$, Proc. 28th Int. Cosmic Ray Conf., Tsukuba, 183-186, 2003.

Ahlers, M.: Anomalous Anisotropies of Cosmic Rays from Turbulent Magnetic Fields, Phys. Rev. Lett., 112, 021101, doi:10.1103/PhysRevLett.112.021101, 2014.

Ahlers, M. and Mertsch, P.: Small-Scale Anisotropies of Cosmic Rays from Relative Diffusion, arXiv:1506.05488 [astro-ph.HE], 2015.

Amenomori, M., Ayabe, S., Cui, S. W., Danzengluobu, Ding, L. K., Ding, X. H., Feng, C. F., Feng, Z. Y., Gao, X. Y., Geng, Q. X., Guo, H. W., He, H. H., He, M., Hibino, K., Hotta, N., Hu, H., Hu, H. B., Huang, J., Huang, Q., Jia, H. Y., Kajino, F., Kasahara, K., Katayose, Y., Kato, C., Kawata, K., Le Labaciren, G. M., Li, J. Y.. Lu, H., Lu, S. L., Meng, X. R., Mizutani, K., Mu, J., Munakata, K., Nagai, A., Nanjo, H., Nishizawa, M., Ohnishi, M., Ohta, I., Onuma, H., Ouchi, T., Ozawa, S., Ren, J. R., Saito, T., Sakata, M., Sasaki, T., Shibata, M., Shiomi, A., Shirai, T., Sugimoto, H., Takita, M., Tan, Y. H., Tateyama, N., Torii, S., Tsuchiya, H., Udo, S., Utsugi, T., Wang, B. S., Wang, H., Wang, X., Wang, Y. G., Wu, H. R., Xue, L., Yamamoto, Y., Yan, C. T., Yang, X. C., Yasue, S., Ye, Z. H., Yu, G. C., Yuan, A. F., Yuda, T., Zhang, H. M., Zhang, J. L., Zhang, N. J., Zhang, X. Y., Zhang, 
Y., Zhang, Y.; Zhaxisangzhu, Zhou, X. X., and Tibet Asy Collaboration: Large-scale sidereal anisotropy of Galactic cosmic-ray intensity observed by the Tibet air shower array, Astrophys. J., 626, L29-L32, 2005.

Antoni, T., Apel, W. D., Badea, A. F., Bekk, K., Bercuci, A., Blümer, H., Bozdog, H., Brancus, I. M., Büttner, C., Daumiller, K., Doll, P., Engel, R., Engler, J., Fessler, F., Gils, H. J., Glasstetter, R., Haungs, A., Heck, D., Hörandel, J. R., Kampert, K.-H., Klages, H. O., Maier, G., Mathes, H. J., Mayer, H. J., Milke, J., Müller, M., Obenland, R., Oehlschläger, J., Ostapchenko, S., Petcu, M., Rebel, H., Risse, A., Risse, M., Roth, M., Schatz, G., Schieler, H., Scholz, J., Thouw, T., Ulrich, H., van Buren, J., Vardanyan, A., Weindl, A., Wochele, J., Zabierowski, J., and KASCADE Collaboration: Large scale cosmic - ray anisotropy with KASCADE, Astrophys. J., 604, 687-692, 2004.

Blasi, P. and Amato, E.: Diffusive propagation of cosmic rays from supernova remnants in the Galaxy, II: anisotropy, J. Cosmol. Astropar. Phys., 11, 011, 2012.

Borriello, E., Maccione, L., and Cuoco, A.: Dark matter electron anisotropy: a universal upper limit, Astropart. Phys., 35, 537546, 2012.

Buesching, I., de Jager, O. C., Potgieter, M. S. and Venter, C.: A Cosmic Ray Positron Anisotropy due to Two Middle-Aged, Nearby Pulsars?, Astrophys. J., 678, L39-L42, doi:10.1086/588465, 2008.

Di Bernardo, G., Evoli, C., Gaggero, D., Grasso, D., Maccione, L., and Mazziotta, M. N.: Implications of the Cosmic Ray Electron Spectrum and Anisotropy measured with Fermi-LAT, Astropart. Phys., 34, 528-538, 2011.

Evoli, C., Gaggero, D., Grasso, D., and Maccione, L.: A common solution to the cosmic ray anisotropy and gradient problems, Phys. Rev. Lett., 108, 211102, 2012.

Gerhardy, P. R. and Clay, R. W.: Southern Hemisphere Cosmic Ray Anisotropy At 10**16-ev, J. Phys. G., 9, 1279-1287, 1983.

Giacinti, G. and Sigl, G.: Local Magnetic Turbulence and TeVPeV Cosmic Ray Anisotropies, Phys. Rev. Lett., 109, 071101, doi:10.1103/PhysRevLett.109.071101, 2012.
Giacinti, G., Kachelriess, M., Semikoz, D. V., and Sigl, G.: Cosmic Ray Anisotropy as Signature for the Transition from Galactic to Extragalactic Cosmic Rays, J. Cosmol. Astropart. Phys., 031, 1207, 2012.

Ginzburg, V. L., Berezinsky, V. S., Bulanov, S. V., and Ptuskin, V. S.: Astrophysics of cosmic rays, North-Holland, Amsterdam, 1990.

Gorski, K. M., Hivon, E., Banday, A. J., Wandelt, B. D., Hansen, F. K., Reinecke, M., and Bartelman, M.: HEALPix - A Framework for high resolution discretization, and fast analysis of data distributed on the sphere, Astrophys. J., 622, 759-771, 2005.

Hillas, A. M.: Can diffusive shock acceleration in supernova remnants account for high-energy galactic cosmic rays?, J. Phys. G., 31, R95-R131, 2005.

Kifune, T., Hara, T., Hatano, Y., Hayashida, N., Honda, M., Kamata, K., Nagano, M., and Nishijima, K.: Anisotropy of Arrival Direction of Extensive Air Showers Observed at Akeno, J. Phys. G., 12, 129-142, 1986.

Linden, T. and Profumo, S.: Probing the Pulsar Origin of the Anomalous Positron Fraction with AMS-02 and Atmospheric Cherenkov Telescopes, Astrophys. J., 772, 18, 2013.

Nagashima, K., Fujimoto, K., Sakakibara, S., Fujii, Z., Ueno, H., Murakami, K., and Morishita, I.: Galactic cosmic ray anisotropy and its modulation in the heliomagnetosphere, inferred from air shower observation at Mt. Norikura, Proc. 21st Int. Cosmic Ray Conf., Adelaide, 180-183, 1990.

Pohl, M. and Eichler, D.: Understanding TeV-band cosmic-ray anisotropy, Astrophys. J., 766, 4, 2013.

Ptuskin, V. S., Jones, F. C., Seo, E. S., and Sina, R.: Effect of random nature of cosmic ray sources Supernova remnants on cosmic ray intensity fluctuations, anisotropy, and electron energy spectrum, Adv. Space Res., 37, 1909-1912, 2006.

Sutherland, M. S., Baughman, B. M., and Beatty, J. J.: CRT: A numerical tool for propagating ultra-high energy cosmic rays through Galactic magnetic field models, Astropart. Phys., 34, 198-204, 2010.

Sveshnikova, L. G., Strelnikova, O. N., and Ptuskin, V. S.: Spectrum and Anisotropy of Cosmic Rays at TeV-PeV-energies and Contribution of Nearby Sources, Astropart. Phys., 33, 50-52, 2013. 